\title{
Horizontal Assembly of Single Nanowire Diode Fabricated by $p$ - $n$ Junction GaN NW Grown by MOCVD
}

\author{
Ji-Hyeon Park, ${ }^{1}$ Suthan Kissinger, ${ }^{2}$ Yong Ho Ra, ${ }^{1}$ Kang San, \\ Min Ji Park, ${ }^{3}$ Kyung-Hwa Yoo, ${ }^{3}$ and Cheul-Ro Lee ${ }^{1}$ \\ ${ }^{1}$ Semiconductor Materials and Process Laboratory, School of Advanced Materials Engineering, \\ Research Center for Advanced Materials Development (RCAMD), Chonbuk National University, Jeonju 561-756, Republic of Korea \\ ${ }^{2}$ Department of General Studies, Physics Group, Jubail University College, Royal Commission for Jubail, Jubail 10074, Saudi Arabia \\ ${ }^{3}$ Department of Physics, Yonsei University, Seoul 120-749, Republic of Korea
}

Correspondence should be addressed to Cheul-Ro Lee; crlee7@jbnu.ac.kr

Received 24 January 2014; Accepted 16 June 2014; Published 10 July 2014

Academic Editor: Alireza Talebitaher

Copyright (C) 2014 Ji-Hyeon Park et al. This is an open access article distributed under the Creative Commons Attribution License, which permits unrestricted use, distribution, and reproduction in any medium, provided the original work is properly cited.

\begin{abstract}
Uniaxially $p-n$ junction gallium nitride nanowires have been synthesized via metal-organic chemical vapor deposition method. Nanowires prepared on $\mathrm{Si}(111)$ substrates were found to grow perpendicular to the substrate, and the transmission electron microscopy studies demonstrated that the nanowires had singlecrystalline structures with a $<0001>$ growth axis. The parallel assembly of the $p-n$ junction nanowire was prepared on a $\mathrm{Si}$ substrate with a thermally grown $\mathrm{SiO}_{2}$ layer. The transport studies of horizontal gallium nitride nanowire structures assembled from $p$ - and $n$-type materials show that these junctions correspond to well-defined $p$ - $n$ junction diodes. The $p$ - $n$ junction devices based on $\mathrm{GaN}$ nanowires suspended over the electrodes were fabricated and their electrical properties were investigated. The horizontally assembled gallium nitride nanowire diodes suspended over the electrodes exhibited a substantial increase in conductance under UV light exposure. Apart from the selectivity to different light wavelengths, high responsivity and extremely short response time have also been obtained.
\end{abstract}

\section{Introduction}

Over the past several years, wide band gap gallium nitride $(\mathrm{GaN})$ nanowires (NWs) have been extensively studied due to their extremely dense logic and unique physical properties with tunable and well-defined atomic composition [1-7]. Recently, single-crystalline semiconductor nanostructures have received great attention due to their superior properties and potential applications in comparison with their bulk form. Among the nanostructured materials, nanowires are very interesting with the charge carrier confined in a onedimensional (1D) space owing to their special configuration.

Successful growth of GaN nanowires on silicon and other mismatched substrates has been demonstrated by several workers [8-11]. The nanowires exhibit significantly reduced defect density due to their large surface-to-volume ratio. A reduced strain distribution in the nanostructures also leads to a weaker piezoelectric polarization field. In addition, the enhanced light collection efficiency $[12,13]$ as well as the highly reduced material consumption [14] with respect to the conventional thin film devices makes nanowires quite attractive for the development of optoelectronic devices. Also, nanoscale control during device fabrication allows systematic exploitation of quantum confinement effects in LEDs [15-17]. It is noted that the luminescence wavelength can be tuned precisely by controlling the dimensions of the nanostructures [18]. Fabricating vertically aligned nanostructures enables the formation of three-dimensional LED architectures, which have a great potential for improved device efficiency due to an enhanced light extraction efficiency [19], increased light emitting active area, and reduced quantum Stark effect [20].

The range of potential applications of semiconductor nanowires keeps increasing in the fields of device miniaturization [21], field-effect transistors [2, 22, 23], photovoltaic cells and light emitting devices [24-27], and so forth. To fabricate nanometer-scale devices, with bottom-up techniques, 
a conventional approach is to start with random dispersion from a nanowire or nanostructure suspension, followed by electrode fabrication at a known nanostructure location on the substrate $[28,29]$. Several experimental techniques have been reported for manipulation of $\mathrm{GaN}$ nanowires into welldefined arrays for integrated devices. A high-brightness $n$ GaN nanowire on $p$-GaN has been synthesized using dielectrophoresis method by Lee et al. [30]. One-dimensional GaN, nanostructure arrays with low defect density are fabricated employing porous anodic alumina films as the template and lead to high-performance devices [31]. The aligned and dislocation free $\mathrm{GaN}$ one-dimensional nanostructures have been fabricated using molecular beam epitaxy (MBE) [32,33], metal-organic chemical vapor deposition (MOCVD) [3437], and hydride vapor-phase epitaxy (HVPE) [38, 39]. Tang et al. [40] have conducted controlled synthesis of a vertically aligned $p$-GaN nanorod array on an $n$-Si substrate by thermal evaporation of $\mathrm{GaCl}_{3}$.

In the study reported here, we have conducted a detailed investigation of the horizontal assembly of $p-n$ junction GaN nanowires directly on $\mathrm{Si}(111)$ substrates by MOCVD. The structural, optical, and electrical characteristics of the nanowires have been measured and are discussed. $p$ - and $n$ type doping of the nanowires is accomplished by introducing trace amounts of $\mathrm{Mg}$ and $\mathrm{Si}$, respectively. This doping capability enables the formation of an epitaxial $p-n$ homojunction, which leads to highly efficient radiative recombination between electrons and holes $[41,42]$. Finally, $p-n$ junction $\mathrm{GaN}$ array diode has been fabricated horizontally with an ensemble of nanowires and the properties of these diodes are also presented. The grown $p-n$ junction GaN nanowires were characterized by field emission scanning electron microscopy (FE-SEM), X-ray diffraction (XRD), photoluminescence (PL), and cathodoluminescence (CL) spectroscopy. The morphology of as-grown nanowires was further analyzed by high resolution transmission electron microscopy (HRTEM). The response time of the $p-n$ junction GaN nanowire was measured by using Nd-YAG laser at the fourth frequency $(266 \mathrm{~nm})$ with $6 \mathrm{~ns}$ Gaussian pulses.

1.1. Materials and Methods. Horizontally aligned $p-n$ junction $\mathrm{GaN}$ nanowires were grown on $\mathrm{Si}(111)$ substrates in a handmade MOCVD system with a horizontal quartz reactor. Before the substrate was loaded into the reactor, it was cleaned by ultrasonic cleaning in acetone and methyl alcohol for $5 \mathrm{~min}$. After that the substrates were overflowed with DI water for $5 \mathrm{~min}$ and etched by HF (2\%) acid for $5 \mathrm{~min}$ to remove the native oxide layer.

To investigate the more complex nanowire structures required for active $p$ - $n$ junction devices, we exploited MOCVD, which is a technique used extensively for the growth of planar GaN-based heterostructures, as a means of $\mathrm{Ga}$ and $\mathrm{NH}_{3}$ reactants as well as silicon and magnesium dopants in a highly controlled and reproducible manner. Trimethylgallium (TMG), ammonia $\left(\mathrm{NH}_{3}\right)$, silane $\left(\mathrm{SiH}_{4}\right.$, $10 \mathrm{ppm}$ diluted in $\mathrm{H}_{2}$ ), and $\mathrm{Cp}_{2} \mathrm{Mg}$ are the precursors for $\mathrm{Ga}, \mathrm{N}$, and $\mathrm{Mg}$, respectively. Hydrogen $\left(\mathrm{H}_{2}\right)$ was used as the carrier gas in the present experiment. Figure 1 illustrates the fabrication flow chart of $p-n$ junction nanowires on
Si(111) substrates after providing several procedures. We have fabricated $n$-GaN:Si nanowire on Si substrate by newly developed two-step growth process. The first stage of the two-step growth process typically begins with a coated $\mathrm{Au}$ film on $\mathrm{Si}(111)$ substrate by ion sputtering system. The $\mathrm{Au}+$ Ga nanodroplets were formed by MOCVD under hydrogen ambient for $10 \mathrm{~min}$. These nanodroplets act as a nucleation seed for the growth of GaN nanowire. After that, the pulsed n$\mathrm{GaN}$ seeds were grown on $\mathrm{Au}+\mathrm{Ga}$ nanodroplets using pulsed flow method. Pulsed $n$-GaN seeds were grown up to 10 pairs at a working pressure and temperature of about 600 torr and $710^{\circ} \mathrm{C}$, respectively. During the pulsed growth mode, group III and group V precursors were introduced alternately in the following sequence: TMG, $3 \mathrm{~min} ; \mathrm{NH}_{3}, 3 \mathrm{~min}$. In the last step, $n-\mathrm{GaN} / p-\mathrm{GaN}$ nanowires were grown on pulsed $n-\mathrm{GaN}$ seeds at $920^{\circ} \mathrm{C}(1$ hour $)$ and $890^{\circ} \mathrm{C}(20 \mathrm{~min})$, respectively, by continuous flow mode. During the continuous flow mode, group III and group V precursors were introduced simultaneously. The $n$-GaN nanowires were grown with the doping of silane gas, which is a critical factor to initiate the vertical nanowire growth. The experiment was carried out for the $\mathrm{Cp}_{2} \mathrm{Mg}$ flow rate of $5 \mathrm{sccm}$ (standard cubic centimeters per minute). The single-crystalline $\mathrm{GaN}$ nanowires were horizontally assembled on a degenerately doped silicon wafer covered with $300 \mathrm{~nm} \mathrm{SiO}_{2}$. The $\mathrm{GaN}$ nanowires were dispersed on the grid using a micropipette. After the evaporation of the ethanol, polymethyl methacrylate (PMMA) was spin coated for $40 \mathrm{sec}$ on the grid at $5000 \mathrm{rpm}$. Using e-beam lithography process the PMMA was removed partially. 5/100 nm thick $\mathrm{Ti} / \mathrm{Pt}$ electrodes were deposited on both ends of the aligned nanowires by DC sputtering method. The remaining PMMA was removed using lift-off technique using acetone.

In our present study, we have intentionally reduced the growth temperature/pressure, so that the drift velocities of the adatoms were increased dramatically. At this stage, the adatoms could move all along the surface of the nanowires to form the sheath structure. Hence in order to induce the smooth growth surface we have changed our growth temperature from $920^{\circ} \mathrm{C}$ to $890^{\circ} \mathrm{C}$. The dopant was changed during the growth procedure in order to obtain the middle point of the nanowire. This process enhances the linear shape of the nanowire and controls the tape-like growth. During the first stage of the growth, we can obtain an $n$ type nanowire in nominally Si doped GaN according to the presence of nitrogen vacancies and/or oxygen impurities. Magnesium doping follows, carried out by supplying $\mathrm{Cp}_{2} \mathrm{Mg}$ during the latter half of the growth, thus leading to $p$-type growth. Finally, the as-prepared $p-n$ junction nanowires were assembled horizontally on a $\mathrm{Cr} / \mathrm{Au}$ metal grid by using ebeam lithography, DC sputter, and lift-off techniques.

The morphology of the $p-n$ junction nanowire arrays was investigated by field emission scanning electron microscopy (FE-SEM/JEOL JSM-6500F, KBSI in Jeonju). Field emission measurements were conducted in a vacuum chamber with a pressure of $1.2 \times 10^{-6} \mathrm{~Pa}$ at room temperature. A rodlike stainless steel probe with $1 \mathrm{~mm}$ diameter and $0.78 \mathrm{~mm}^{2}$ in area was used as anode. The sample was used as cathode. The spacing between the two electrodes is $100 \mu \mathrm{m}$. A ballast resistor of $10 \mathrm{MV}$ was used to protect the apparatus 


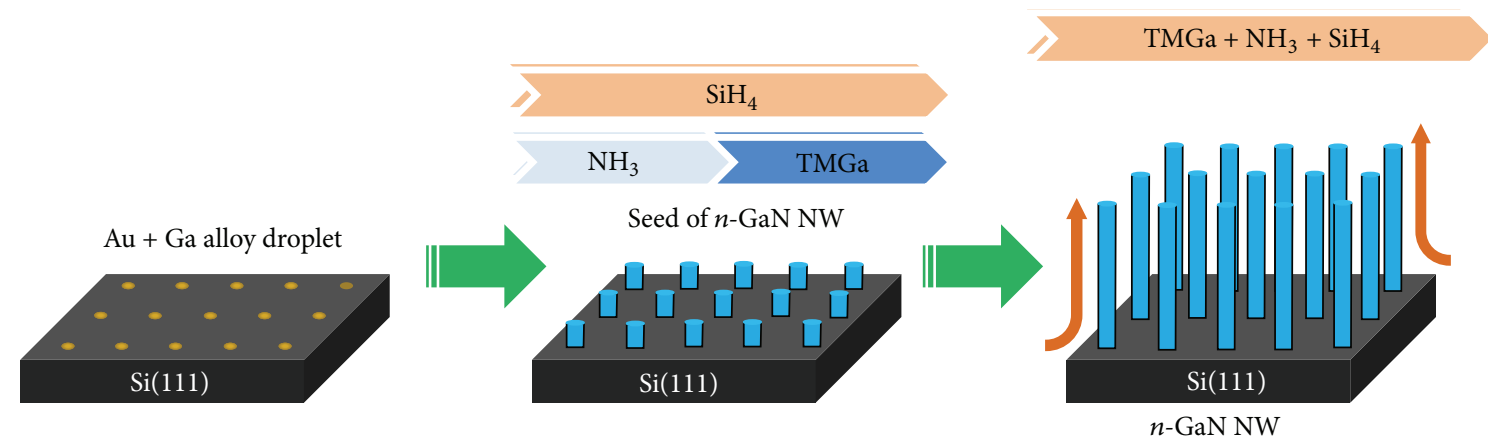

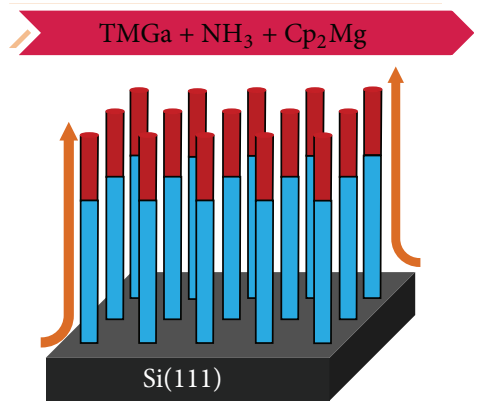

Pulsed growth of $p$-GaN NWs
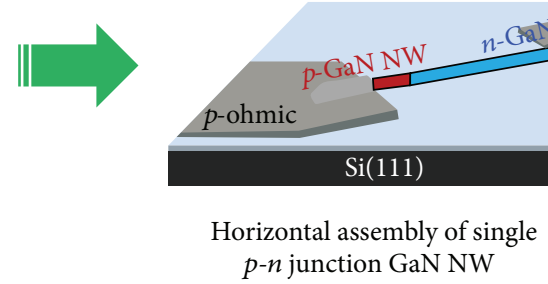

FIGURE 1: Schematic illustration of horizontally assembled single $p$ - $n$ junction nanowire diode fabricated on $\mathrm{SiO}_{2} / \mathrm{Si}$ substrates. (i) $\mathrm{Au}+$ Ga droplets formation on $\mathrm{Si}(111)$ substrate, (ii) $n$ - $\mathrm{GaN}$ seed formation at $710^{\circ} \mathrm{C}$, (iii) $n$-GaN/Si nanowire formation at $920^{\circ} \mathrm{C}(1 \mathrm{hour}$ ), (iv) formation of $p-\mathrm{GaN} / \mathrm{Mg}$ nanowire at $850^{\circ} \mathrm{C}(20 \mathrm{~min})$, and $(\mathrm{v})$ horizontal assembly of $p$ - $n$ junction nanowire using e-beam lithography and lift-off techniques.

against short circuiting. The optical properties of the asgrown nanowire arrays were characterized by photoluminescence (PL) spectrum using a $325 \mathrm{~nm}$ He-Cd laser and a cathodoluminescence (CL) attached to the FE-SEM system. Microstructure and single crystallinity of the nanowires were confirmed by the high resolution transmission electron microscopy (HR-TEM) and selected area electron diffraction (SAED) analysis. The current-voltage $(I / V)$ measurements for the horizontally assembled nanowires were carried out under different illumination environments.

\section{Results of the Experimentation}

Figure 2(a) shows the tilt-view FE-SEM images of $n$-GaN:Si nanowires on $n$-GaN seeds. In the early stage of the $n$-GaN seed growth, Au catalyst is known to play an important role to nucleate the seed growth. But at high temperature it is difficult to nucleate $n-\mathrm{GaN}: \mathrm{Si}$ nanowires with Au catalyst alone. The number of seed pairs is a crucial factor in determining the dimension and density of nanowires. The density of $n$ GaN nanowires was increased monotonically with increase in number of seed pairs. Moreover, it is observed from the figure that large-scale, vertically, or slantingly aligned GaN nanowires were uniformly grown in high density on the $\mathrm{Si}(111)$ substrate and have clean surface. Notably, Au clusters after supporting the formation of GaN nuclei may leave the location owing to the high diffusion rate of $\mathrm{Au}$ atoms at high growth temperatures $[43,44]$. Most probably, small Au clusters react with the $\mathrm{GaN}$ layer chemically during the wire growth. Therefore, in this case the Au particle catalyzing the $\mathrm{GaN}$ nanowire growth remains neither at the root nor at the tip after extended nanowire growth period. From the FE-SEM data, the average diameters of the nanowires were about $300-$ $400 \mathrm{~nm}$ and their lengths are mainly approximately $4 \mu \mathrm{m}$. The diameters of the $n$-GaN nanowires were observed to be uniform from the top to the bottom.

Figure 2(b) shows panchromatic CL spectra of $n$-GaN nanowires grown on $n$-GaN seed. The emitted CL was collimated by mirrors located near the sample, transmitted through a fused-silica window in the SEM, and refocused onto the entrance slit of a $0.34 \mathrm{~mm}$ spectrograph, with a 600 line $/ \mathrm{mm}$ grating and entrance slit width of $0.05 \mathrm{~mm}$. The spectra were recorded by a computer-controlled, nitrogencooled CCD camera. The wavelength resolution was $0.29 \mathrm{~nm}$, which is equivalent to an energy resolution of $2.3 \times 10^{-4} \mathrm{E}^{2} \mathrm{eV}$, where $E$ is photon energy. The CL experiments were carried out at a temperature of $300 \mathrm{~K}$. The GaN signature in the $290-$ $360 \mathrm{~nm}$ wavelength range was used to optimize the CL signal. The applied accelerating voltage $\left(V_{a}\right)$ and beam current $\left(I_{b}\right)$ are $5 \mathrm{kV}$ and $1000 \mathrm{pA}$, respectively. The slit widths were set at $1 \mathrm{~mm}$, which is the optimum width for achieving high count rates. From the CL spectra we could observe that the $n$-GaN:Si nanowires show a near-band-edge emission (NBE) centered at $370 \mathrm{~nm}$ in the ultraviolet range of the spectrum due to the increased excitation rates associated with CL. We could not observe any deep-level (yellow) CL for the $n$-GaN nanowires arising either directly from the atomic structure of the dislocations or associated with the clustering of native 


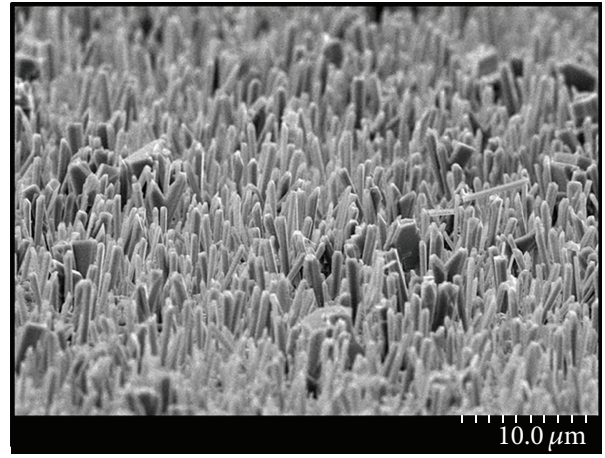

(a)

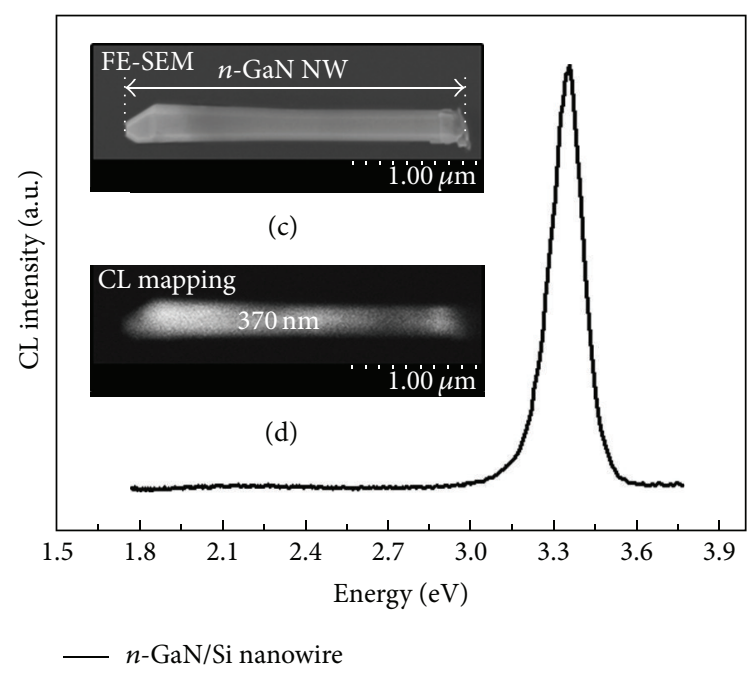

(b)

FIGURE 2: Tilt-view FE-SEM image of (a) $n$-GaN/Si nanowires fabricated on Si(111) substrate, (b) CL spectra of $n$-GaN/Si nanowires, (c) FE-SEM image of single $n$-GaN/Si nanowire, and (d) CL mapping image at $370 \mathrm{~nm}$.

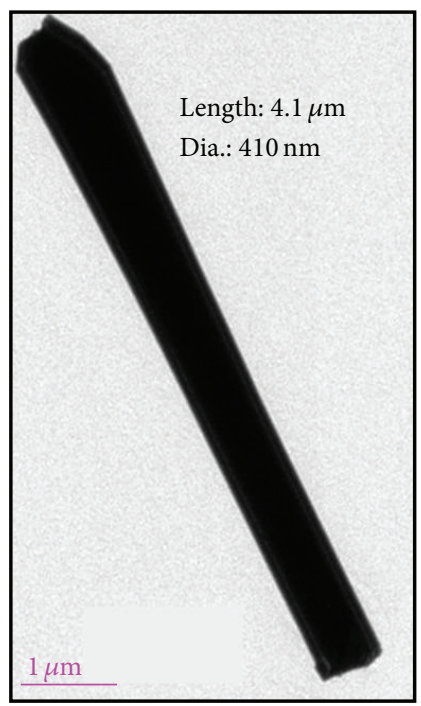

(a)

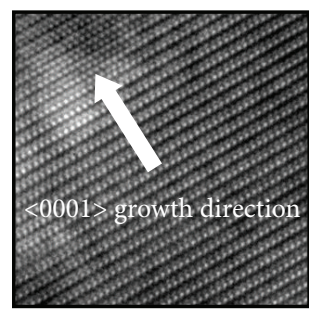

(b)

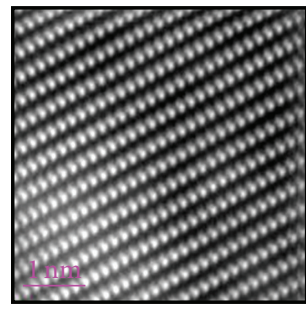

(d)

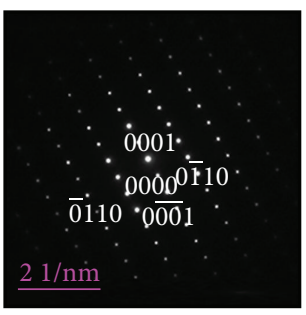

(c)

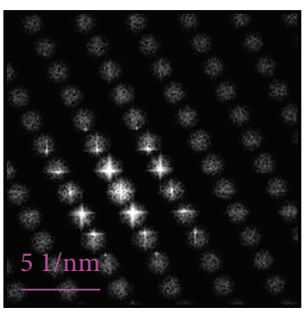

(e)

FIGURE 3: HR-TEM image of the $n$-GaN:Si NWs grown on pulsed $u$-GaN seed: (a) single $n$-GaN:Si NW, (b) lattice structure of $n$-GaN:Si NWs, (c) SAED pattern of the $n$-GaN NW:Si, (d) the lattice image obtained by inverse fast fourier transform (IFFT), and (e) fast fourier transform image (FFT).

point defects (vacancies, interstitials) [45]. From this data, one can understand that the grown $n$-GaN:Si nanowires were high quality with lower defect. Figure 2(c) shows a FE-SEM image of a single $n$-GaN:Si nanowire and its corresponding CL mapping received at wavelength $370 \mathrm{~nm}$ is presented in Figure 2(d). The wavelength $370 \mathrm{~nm}$ is corresponding to the maximum peak energy of the band emission. From the CL image, it is observed that the $370 \mathrm{~nm}$ emission is quite uniform throughout the GaN structure which appeared as white color. From Figure 2(d), one can also see that the emission intensities are quite uniform, which might be related to the homogenous distribution of strain within the selforganized $n$-GaN nanowires.
Figure 3 shows bright field and high resolution TEM images as well as selected area diffraction (SAED) pattern. Figure 3(a) shows the HR-TEM image of a single $n$-GaN:Si nanowire. The stacking of hexagonal basal planes along the growth orientation of the wire and the $<0001>$ direction can be seen in the high resolution mode. The GaN nanowire exhibits a $2 \mathrm{H}$ hexagonal structure. Figure $3(\mathrm{~b})$ shows a highmagnification TEM image of $n$-GaN nanowire. The clear lattice fringes confirmed in the HRTEM image indicate that the nanowire is hexagonal single-crystalline structure without defect or secondary phases. This is consistent with wurtzite $\mathrm{GaN}$ structure and corresponds to the lattice spacing between the $\left(\begin{array}{llll}0 & 0 & 0 & 1\end{array}\right)$ planes in the $\mathrm{GaN}$ phase. Figure 3(c) reveals 


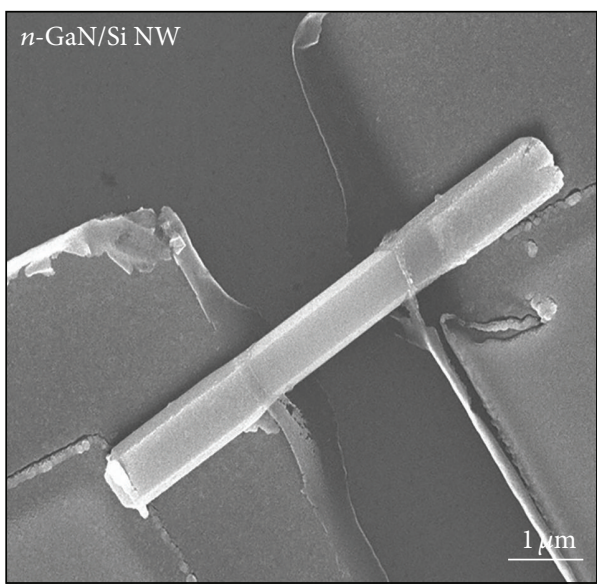

(a)

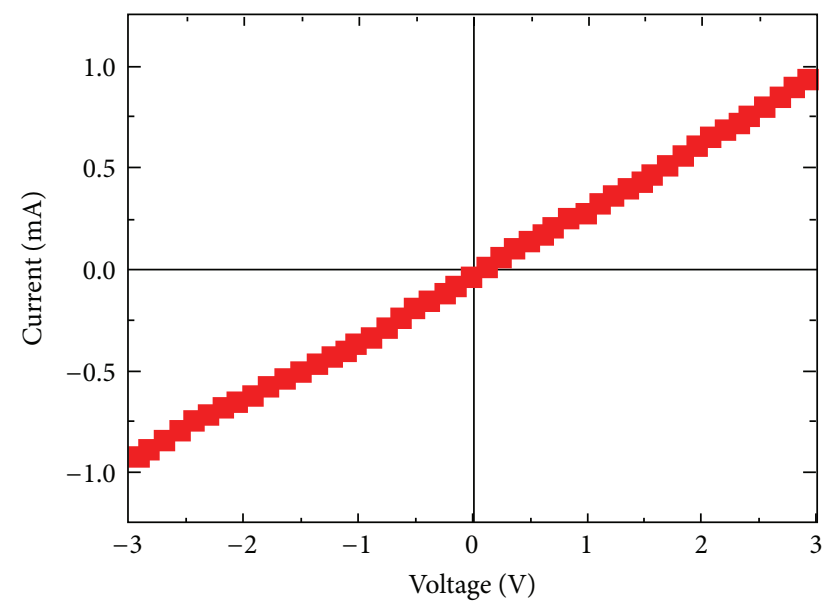

(b)

FIGURE 4: (a) FE-SEM image of horizontally assembled $n$-GaN/Si nanowire, (b) the current-voltage measurements of $n$-GaN/Si nanowire.

the representative SAED pattern, with the (1100), (0001), (0001), and (1100) diffraction spots. These regular diffraction spots demonstrated that $n$-GaN nanowires grew along a $<0001>$ direction. The lattice structure shown in Figure 3(d) represents the IFFT image of high resolution. Such images clearly show the (0001) atomic planes perpendicular to the nanowire axis. Figure 3(e) represents the corresponding FFT pattern of the HRTEM image that indicates the $n$ $\mathrm{GaN}$ nanowires are preferentially oriented along the $c$-axis direction, as well, and are indexed to the reflections of the wurtzite structure.

Figure 4(a) shows the horizontal assembly of single $n$ $\mathrm{GaN}: \mathrm{Si}$ nanowire. The single-crystalline $n$-GaN nanowires fabricated by two-step growth process were horizontally assembled on a degenerately doped silicon wafer covered with $300 \mathrm{~nm} \mathrm{SiO}_{2}$. In the next process, 5/50 nm thick $\mathrm{Cr} / \mathrm{Au}$ metal grid was formed by e-beam lithography, e-beam evaporator, and lift-off techniques. In order to disperse the $n$-GaN nanowires, Si substrate was immersed in ethanol. The substrate immersed in ethanol was sonicated for $5 \mathrm{~min}$. The $n$-GaN nanowires were dispersed on the grid using a micropipette. After the evaporation of the ethanol, PMMA was spin coated for $40 \mathrm{sec}$ on the grid at $5000 \mathrm{rpm}$. Using ebeam lithography process the PMMA was removed partially. $5 / 100 \mathrm{~nm}$ thick Ti/Pt electrodes were deposited on both ends of the aligned nanowires by DC sputtering method. The $I / V$ characteristics of the single $n-\mathrm{GaN}$ nanowire suspended over the electrodes are shown in Figure 4(b). The $I / V$ curve of the $n$-GaN nanowire shows obvious ohmic behaviour of the fabricated nanowire. The linear $I / V$ curve for the fabricated $\mathrm{GaN}$ nanowire reveals that good ohmic contacts have been obtained.

Figure 5 shows the FE-SEM image of a single $p$ - $n$ junction nanowire. It is observed from the figure that the $n-\mathrm{GaN}$ and $p$-GaN growth regions are not distinguishable from each other due to the smooth morphology. This diverse growth rate is due to the reduction in temperature from $920^{\circ} \mathrm{C}$ to $890^{\circ} \mathrm{C}$. Distinctly different from the morphology of $n$-doped $\mathrm{GaN}$ nanowires, $p$-doped $\mathrm{GaN}$ nanowires exhibit smooth morphology throughout the wire with uniform diameter along the length of the nanowire, as observed in SEM image (Figure 5(a)). The $p$-doping leads to a clear morphology evolution from the smooth to the rough one, and thus the aspect ratio of $\mathrm{GaN}$ nanowires increased greatly. Obviously we could observe that the $p$-doping has resulted in the formation of some sharp tips protruding from the GaN nanowire surface (Figure 5(a)). In fact it has been demonstrated that the morphology and geometrical shape of nanostructures are quite sensitive to the adding of foreign impurities even though their content is less than the limit of X-ray energy dispersive spectrometer (EDS) measurement. The introduction of doping elements may affect the growth behaviour or thermodynamics of $\mathrm{GaN}$ nanostructures and leads to the changes of morphology and geometry shape. Figures 5(b) and 5(c) represent the CL spectra measured from the $n$ $\mathrm{GaN}: \mathrm{Si} / p-\mathrm{GaN}: \mathrm{Mg}$ regions. The emissions of both regions were centered at $370 \mathrm{~nm}$ and $436 \mathrm{~nm}$, respectively. The CL peak of $p-\mathrm{GaN}: \mathrm{Mg}$ region is smooth corresponding to the $n$ GaN:Si region without any defect luminescence. The major luminescence at $436 \mathrm{~nm}$ was demonstrated to be contributed uniformly from the whole $p-\mathrm{GaN}: \mathrm{Mg}$ nanowire region. These emissions originated from the transition between the conduction band and $\mathrm{Mg}$-related acceptors. This wavelength is consistent with band-edge emission from a $p$-GaN:Mg structure.

Figure 6(a) represents the FE-SEM image of the horizontally assembled GaN nanowire $p$ - $n$ junction device structure. The current-voltage $(I / V)$ measurements with the $\mathrm{GaN}$ nanowire $p-n$ junction diode were carried out under different illumination environment. Our experiment involved a double-wavelength handheld UV lamp of 254 and $365 \mathrm{~nm}$ in fixed wavelength at a distance of approximately $3 \mathrm{~cm}$ away from the nanodiode. The nanostructured device was kept under practical conditions, that is, in air, at room temperature, and under indoor incandescent light during the measurements. The power density was $0.3 \mathrm{~mW} / \mathrm{cm}^{2}$ for both UV wavelengths. Figure 6(b) shows three $I / V$ curves plotted at gate voltage $\left(V_{g}\right)=0 \mathrm{~V}$ under incandescent light and 


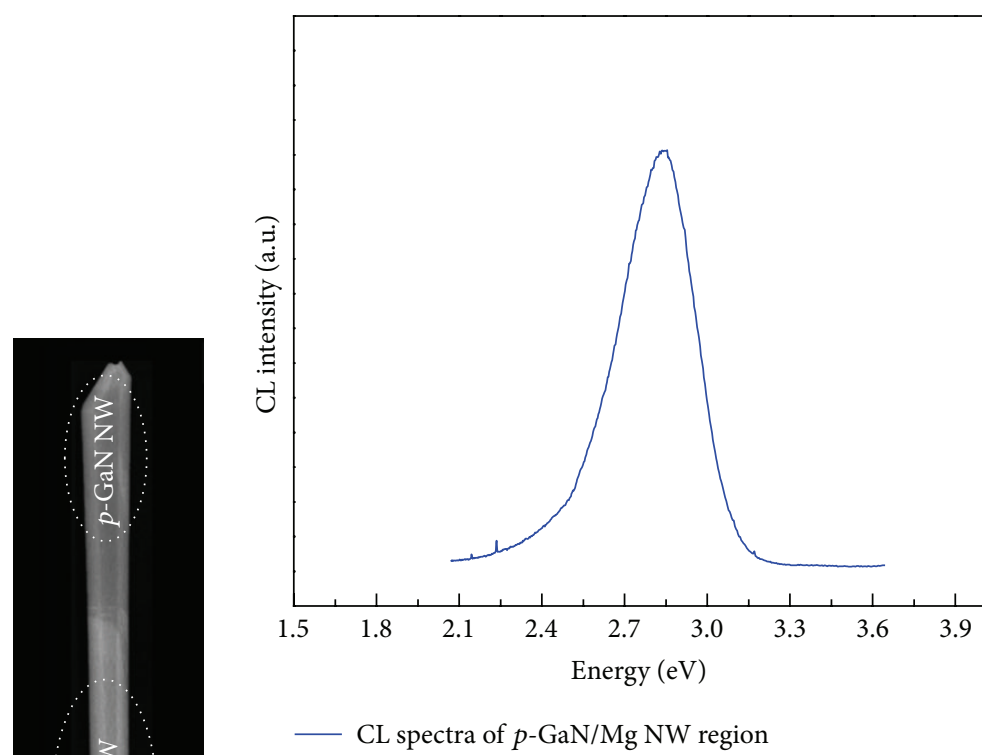

(b)

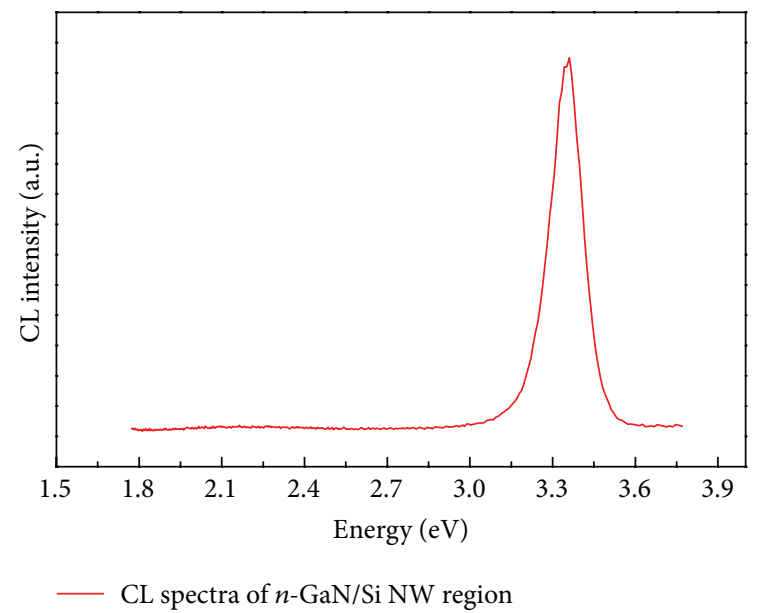

(a)

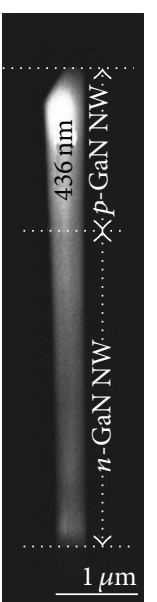

(c)

(d)

Figure 5: FE-SEM image of (a) $p$ - $n$ junction GaN nanowire, (b) and (c) the CL spectrum and mapping (436 $\mathrm{nm}$ ) taken at $p$-GaN/Mg region, and (d) the CL spectrum taken at $n-\mathrm{GaN} / \mathrm{Si}$ region.

upon exposure to UV light at wavelengths of 254 and $365 \mathrm{~nm}$, respectively. Enhanced conduction behaviour was observed for UV illumination of both the wavelengths; nevertheless, the magnitude of both wavelengths differed significantly. The observed data clearly exhibits that under UV exposure the nanowire conductivity increases greatly. The enhanced conductivity under UV light illumination is attributed to the excess photogenerated carriers in the semiconducting nanowire. We have also observed that a higher power density of UV light produces more carriers for conduction. The UV light with a wavelength of $254 \mathrm{~nm}$ has photon energy of $4.88 \mathrm{eV}$, which is sufficient enough to excite electrons across the $3.4 \mathrm{eV}$ bandgap of GaN. Furthermore, the energy of UV light with a wavelength of $365 \mathrm{~nm}$ corresponds to $3.39 \mathrm{eV}$, which is slightly lower than the GaN bandgap. On the other hand, there are still some photons that exist with higher energies due to the nonzero spectrum width, which can generate electron-hole pairs and thus increase the carrier combination of the nanowire. Figure 7 (a) shows the spectral response curve of the $p-n$ junction device structure at zero bias. Spectral photoresponse measurement was done in the 250-500 nm range with a $150 \mathrm{~W}$ Xenon arc lamp light source and a Jobin-Yvon H10-UV monochromator. The monochromated output light was coupled into a multimode UV fiber by which the sample was illuminated. Calibration of the light source output was carried out by using a calibrated Si photodetector and a Newport 1835-C optical power meter. The responsivity was quite flat over the band gap with an abrupt cutoff wavelength at $365 \mathrm{~nm}$. The maximum responsivity value was $0.17 \mathrm{~A} / \mathrm{W}$ around $250 \mathrm{~nm}$. An UV/visible contrast of more than three orders of magnitude was obtained. This indicates that the GaN $p$ - $n$ junction diode has good spectral 


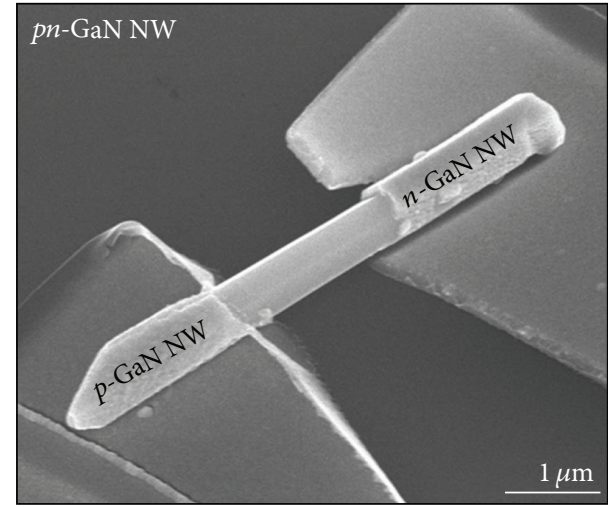

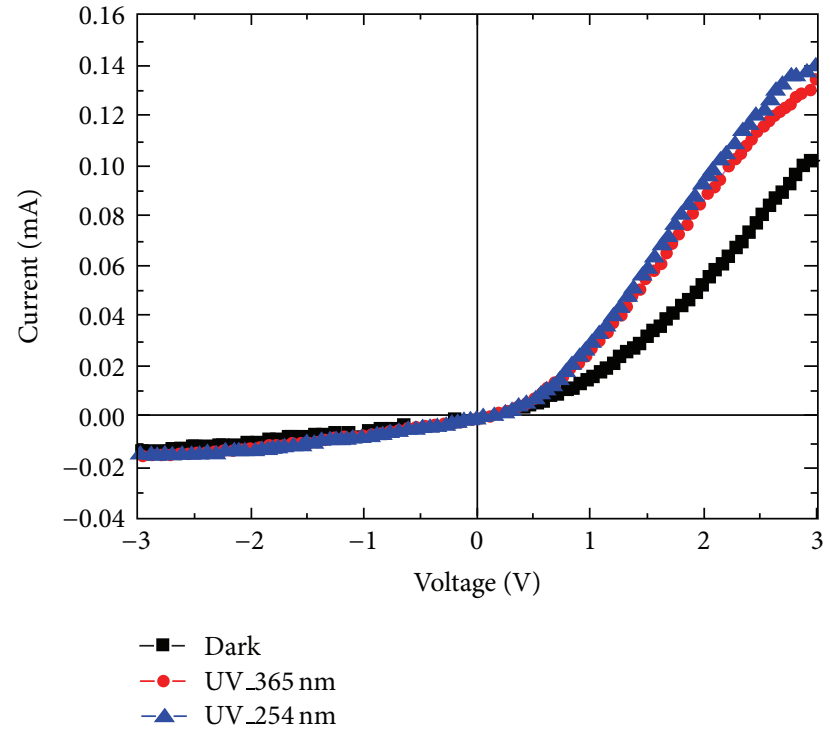

(b)

Figure 6: FE-SEM image of (a) horizontally assembled $p$ - $n$ junction diode by single GaN nanowire, (b) the $I / V$ measurements of GaN nanowire device in the dark and under UV illumination (wavelength $\lambda=365$ and $254 \mathrm{~nm}$ ).

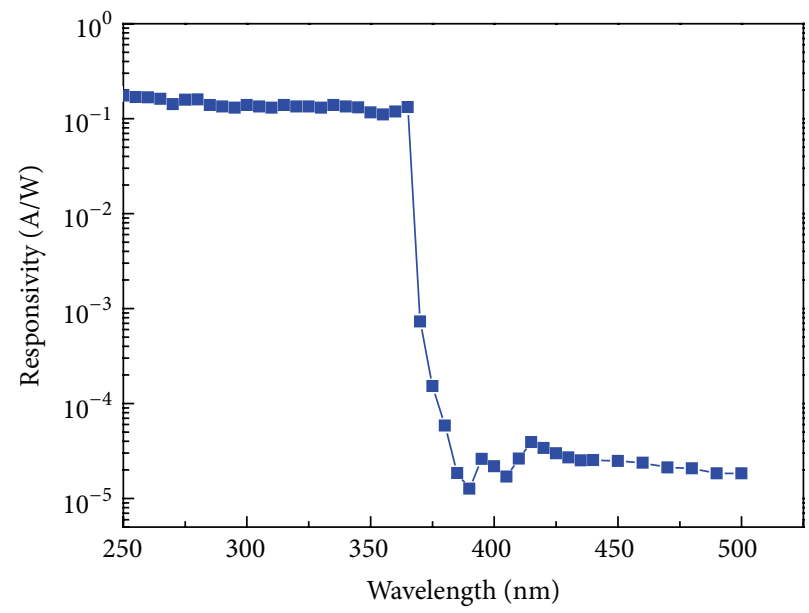

(a)

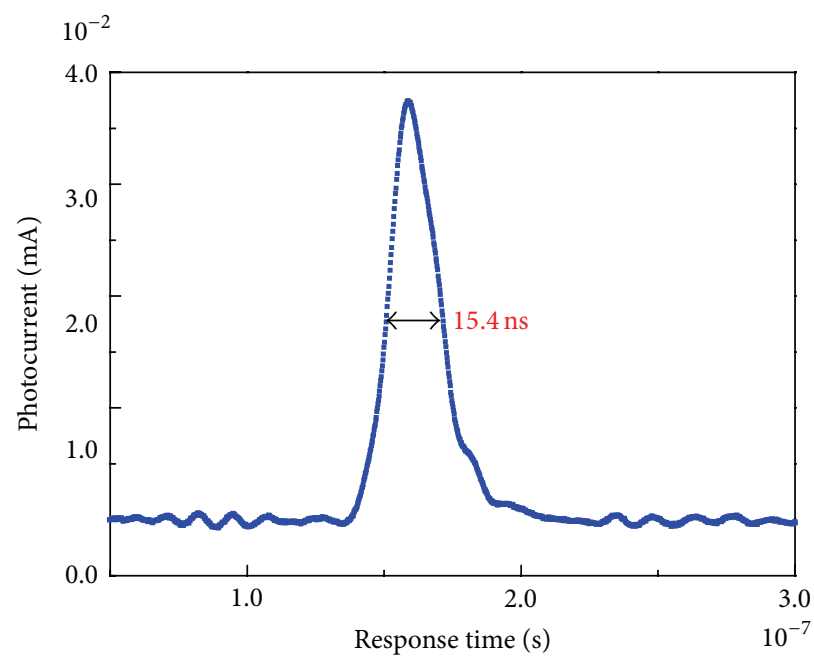

(b)

Figure 7: (a) The responsivity and (b) response time spectra for the single $p-n$ junction GaN nanowire grown on Si(111) substrate.

selectivity. Figure 7(b) shows the response time for the $p-n$ junction single GaN nanowire. The response time of the $p$ $n$ junction $\mathrm{GaN}$ nanowire was measured by using the fourth frequency of Nd-YAG laser $(266 \mathrm{~nm})$ with $6 \mathrm{~ns}$ Gaussian pulses. The $p-n$ junction $\mathrm{GaN}$ nanowire shows a response time with a full width at half maximum (FWHM) of $15.4 \mathrm{~ns}$ under nonbiased condition. The device was Measured at zero bias, but resulted a very fast response time. The response time is significantly faster than the $118 \mathrm{~ns}$ for $n$-GaN Schottky device [46]. It clearly shows that the perturbation induces an electric field along the nanowire that enhances the collection of photogenerated carriers with higher photo response.

\section{Conclusions}

In summary, horizontal assembly of $p$ - $n$ junction singlecrystalline $\mathrm{GaN}$ nanowires has been fabricated via metalorganic chemical vapor deposition method using $\mathrm{Mg}$ as the $p$-type dopant. Nanowires prepared on $\mathrm{Si}(111)$ substrates grow epitaxially with a (002) direction. The prepared GaN nanowires show excellent rectification properties. $n$-doped $\mathrm{GaN}$ nanowires fabricated under similar conditions invariably produce linear current-voltage curves, suggesting that the observed rectification comes from the $p-n$ junction. In addition to that, the transport studies of GaN nanowire structures assembled horizontally from $p$-type and $n$-type 
materials show that the nanoscale junctions behave as the well-defined $p$ - $n$ junction diodes. The photoresponse properties reveal that the nanodiode fabricated in our present study exhibits advantages in high responsivity and response time. And also, it can be concluded that this nanowire photodetector having low dislocation density will show higher sensitivity than the device fabricated by GaN epitaxial layer having high defect density in missile detection and flame sensing applications.

\section{Conflict of Interests}

The authors declare that there is no conflict of interests regarding the publication of this paper.

\section{Acknowledgments}

This research was supported by Basic Science Research Program through the National Research Foundation of Korea (NRF) funded by the Ministry of Education (BRL no. 20100019626). This research was financially supported by the Ministry of Education, Science and Technology (MEST) and National Research Foundation of Korea (NRF) through the Human Resource Training Project for Regional Innovation. This research was financially supported by the Ministry of Knowledge Economy (MKE), Korea Institute for Advancement of Technology (KIAT), and Honam Leading Industry Office through the Leading Industry Development for Economic Region.

\section{References}

[1] F. A. Ponce and D. P. Bour, "Nitride-based semiconductors for blue and green light-emitting devices," Nature, vol. 386, no. 6623, pp. 351-359, 1997.

[2] J. Xiang, W. Lu, Y. Hu, Y. Wu, H. Yan, and C. M. Lieber, "Ge/Si nanowire heterostructures as high-performance fieldeffect transistors," Nature, vol. 441, no. 7092, pp. 489-493, 2006.

[3] S. J. Pearton, J. C. Zolper, R. J. Shul, and F. Ren, "GaN: processing, defects, and devices," Journal of Applied Physics, vol. 86, no. 1, pp. 1-15, 1999.

[4] D. Wang, B. A. Sheriff, and J. R. Heath, "Complementary symmetry silicon nanowire logic: power-efficient inverters with gain," Small, vol. 2, no. 10, pp. 1153-1158, 2006.

[5] P. Waltereit, O. Brandt, A. Trampert et al., "Nirtride semiconductors free of electrostatic fields for efficient white lightemitting diodes," Nature, vol. 406, no. 6798, pp. 865-868, 2000.

[6] L. Polenta, M. Rossi, A. Cavallini et al., "Investigation on localized states in GaN nanowires," ACS Nano, vol. 2, no. 2, pp. 287-292, 2008.

[7] E. Lai, W. Kim, and P. Yang, "Vertical nanowire array-based light emitting diodes," Nano Research, vol. 1, no. 2, pp. 123-128, 2008.

[8] J. Miao, W. Guo, N. Lu et al., "Single InAs nanowire roomtemperature near-infrared photodetectors," ACS Nano, vol. 8, no. 4, pp. 3628-3635, 2014.

[9] A. Afal, S. Coskun, and H. E. Unalan, "All solution processed, nanowire enhanced ultraviolet photodetectors," Applied Physics Letters, vol. 102, no. 4, Article ID 043503, 2013.
[10] T. Kuykendall, P. Ulrich, S. Aloni, and P. Yang, "Complete composition tunability of InGaN nanowires using a combinatorial approach," Nature Materials, vol. 6, no. 12, pp. 951-956, 2007.

[11] R. Armitage and K. Tsubaki, "Multicolour luminescence from InGaN quantum wells grown over GaN nanowire arrays by molecular-beam epitaxy," Nanotechnology, vol. 21, no. 19, Article ID 195202, 2010.

[12] E. Garnett and P. Yang, "Light trapping in silicon nanowire solar cells," Nano Letters, vol. 10, no. 3, pp. 1082-1087, 2010.

[13] S. L. Diedenhofen, G. Vecchi, R. E. Algra et al., "Broadband and omnidirectional antireflection coatings based on semiconductor nanorods," Advanced Materials, vol. 21, no. 9, pp. 973-978, 2009.

[14] J. Kupec, R. L. Stoop, and B. Witzigmann, "Light absorption and emission in nanowire array solar cells," Optics Express, vol. 18, no. 26, pp. 27589-27605, 2010.

[15] R. Ghosh and D. Basak, "Quantum confinement of excitons in dendrite-like GaN nanowires," Journal of Applied Physics, vol. 98, no. 8, Article ID 086104, 2005.

[16] J. Ristić, C. Rivera, E. Calleja, S. Fernández-Garrido, M. Povoloskyi, and A. Di Carlo, "Carrier-confinement effects in nanocolumnar GaN AlxGal-xN quantum disks grown by molecular-beam epitaxy," Physical Review B-Condensed Matter and Materials Physics, vol. 72, no. 8, Article ID 085330, 2005.

[17] H. S. Chen, D. Yeh, Y. Lu et al., "Strain relaxation and quantum confinement in InGaN/GaN nanoposts," Nanotechnology, vol. 17, no. 5, pp. 1454-1457, 2006.

[18] H. Im, Y. S. Park, S.-K. Lee, Y.-H. Cho, and R. A. Taylor, "Microand time-resolved photoluminescence in $\mathrm{GaN}$ nanorods with different diameters," Journal of the Korean Physical Society, vol. 57, no. 4, pp. 756-759, 2010.

[19] S. J. An, J. H. Chae, G. Yi, and G. H. Park, "Enhanced light output of GaN-based light-emitting diodes with $\mathrm{ZnO}$ nanorod arrays," Applied Physics Letters, vol. 92, no. 12, Article ID 121108, 2008.

[20] C. Wang, L. Chen, G. Chen et al., "GaN nanorod light emitting diode arrays with a nearly constant electroluminescent peak wavelength," Optics Express, vol. 16, no. 14, pp. 10549-10556, 2008.

[21] Y. Huang, X. F. Duan, Y. Cui, L. J. Lauhon, K. H. Kim, and C. M. Lieber, "Logic gates and computation from assembled nanowire building blocks," Science, vol. 294, no. 5545, pp. 1313-1317, 2001.

[22] Y. Cui, Z. Zhong, D. Wang, W. U. Wang, and C. M. Lieber, "High performance silicon nanowire field effect transistors," Nano Letters, vol. 3, no. 2, pp. 149-152, 2003.

[23] A. L. Vallett, S. Minassian, P. Kaszuba, S. Datta, J. M. Redwing, and T. S. Mayer, "Fabrication and characterization of axially doped silicon nanowire tunnel field-effect transistors," Nano Letters, vol. 10, no. 12, pp. 4813-4818, 2010.

[24] A. Hagfeld and M. Grätzel, "Light-induced redox reactions in nanocrystalline systems," Chemical Reviews, vol. 95, no. 1, pp. 49-68, 1995.

[25] B. Tian, X. Zheng, T. J. Kempa et al., "Coaxial silicon nanowires as solar cells and nanoelectronic power sources," Nature, vol. 449, no. 7164, pp. 885-889, 2007.

[26] M. R. Lee, R. D. Eckert, K. Forberich, G. Dennler, C. J. Brabec, and R. A. Gaudiana, "Solar power wires based on organic photovoltaic materials," Science, vol. 324, no. 5924, pp. 232-235, 2009.

[27] H. Zhang, Y. Li, Q. Tang, L. Liu, and Z. Zhou, "First-principles studies on structural and electronic properties of GaN-AlN heterostructure nanowires," Nanoscale, vol. 4, no. 4, pp. 10781084, 2012. 
[28] Y. Xia, P. Yang, Y. Sun et al., "One-dimensional nanostructures: synthesis, characterization, and applications," Advanced Materials, vol. 15, no. 5, pp. 353-389, 2003.

[29] T. Kuykendall, P. Pauzauskie, S. Lee, Y. Zhang, J. Goldberger, and P. Yang, "Metalorganic chemical vapor deposition route to $\mathrm{GaN}$ nanowires with triangular cross sections," Nano Letters, vol. 3, no. 8, pp. 1063-1066, 2003.

[30] S.-K. Lee, T.-H. Kim, K.-C. Choi, and P. Yang, "High-brightness gallium nitride nanowire UV-blue light emitting diodes," Philosophical Magazine, vol. 87, no. 14-15, pp. 2105-2115, 2007.

[31] P. Deb, H. Kim, Y. Qin et al., "GaN nanorod schottky and pn junction diodes," Nano Letters, vol. 6, no. 12, pp. 2893-2898, 2006.

[32] R. Calarco, R. J. Meijers, R. K. Debnath, T. Stoical, E. Sutter, and H. Lüth, "Nucleation and growth of GaN nanowires on $\mathrm{Si}(111)$ performed by molecular beam epitaxy," Nano Letters, vol. 7, no. 8, pp. 2248-2251, 2007.

[33] Y. S. Park, C. M. Park, D. J. Fu, T. W. Kang, and J. E. Oh, "Photoluminescence studies of $\mathrm{GaN}$ nanorods on $\mathrm{Si}$ (111) substrates grown by molecular-beam epitaxy," Applied Physics Letters, vol. 85, no. 23, pp. 5718-5720, 2004.

[34] J. Goldberger, R. He, Y. Zhang et al., "Single-crystal gallium nitride nanotubes," Nature, vol. 422, no. 6932, pp. 599-602, 2003.

[35] Y. Wang, K. Zang, S. Chua, M. S. Sander, S. Tripathy, and C. G. Fonstad, "High-density arrays of InGaN nanorings, nanodots, and nanoarrows fabricated by a template-assisted approach," Journal of Physical Chemistry B, vol. 110, no. 23, pp. 11081-11087, 2006.

[36] Y. M. Lee, R. Navamathavan, K. Song et al., "Bicrystalline GaN nanowires grown by the formation of PtGa solid solution nanodroplets on Si(111) using MOCVD," Journal of Crystal Growth, vol. 312, no. 16-17, pp. 2339-2344, 2010.

[37] P. Deb, H. Kim, V. Rawat et al., "Faceted and vertically aligned GaN nanorod arrays fabricated without catalysts or lithography," Nano Letters, vol. 5, no. 9, pp. 1847-1851, 2005.

[38] H.-M. Kim, D. S. Kim, Y. S. Park, D. Y. Kim, T. W. Kang, and K. S. Chung, "Growth of GaN nanorods by a hybrid vapour phase epitaxy method," Advanced Materials, vol. 14, no. 13-14, pp. 991993, 2002.

[39] H.-M. Kim, T. W. Kang, and K. S. Chung, "Growth of GaN nanorods by a hydride vapor phase epitaxy method," Advanced Materials, vol. 15, pp. 567-569, 2003.

[40] Y. B. Tang, Z. H. Chen, H. S. Song et al., "Vertically aligned p-type single-crystalline GaN nanorod arrays on n-type $\mathrm{Si}$ for heterojunction photovoltaic cells," Nano Letters, vol. 8, no. 12, pp. 4191-4195, 2008.

[41] H. Amano, M. Kito, K. Hiramatsu, and I. Akasaki, "P-type conduction in Mg-doped GaN treated with low-energy electron beam irradiation (LEEBI)," Japanese Journal of Applied Physics, vol. 28, pp. L2112-L2114, 1989.

[42] M. E. Lin, B. N. Sverdlov, and H. Morkoç, “Thermal stability of GaN investigated by low-temperature photoluminescence spectroscopy," Applied Physics Letters, vol. 63, no. 26, pp. 36253627, 1993.

[43] T. Stoica, E. Sutter, R. J. Meijers et al., "Interface and wetting layer effect on the catalyst-free nucleation and growth of $\mathrm{GaN}$ nanowires," Small, vol. 4, no. 6, pp. 751-754, 2008.

[44] L. Qin, C. Xue, Y. Duan, and L. Shi, "Synthesis and characterization of glomerate GaN nanowires," Nanoscale Research Letters, vol. 4, no. 6, pp. 584-587, 2009.
[45] X. Chen, J. Li, Y. Cao et al., "Straight and smooth GaN nanowires," Advanced Materials, vol. 12, no. 19, pp. 1432-1434, 2000.

[46] J. M. van Hove, R. Hickman, J. J. Klaassen, P. P. Chow, and P. P. Ruden, "Ultraviolet-sensitive, visible-blind GaN photodiodes fabricated by molecular beam epitaxy," Applied Physics Letters, vol. 70, no. 17, pp. 2282-2284, 1997. 

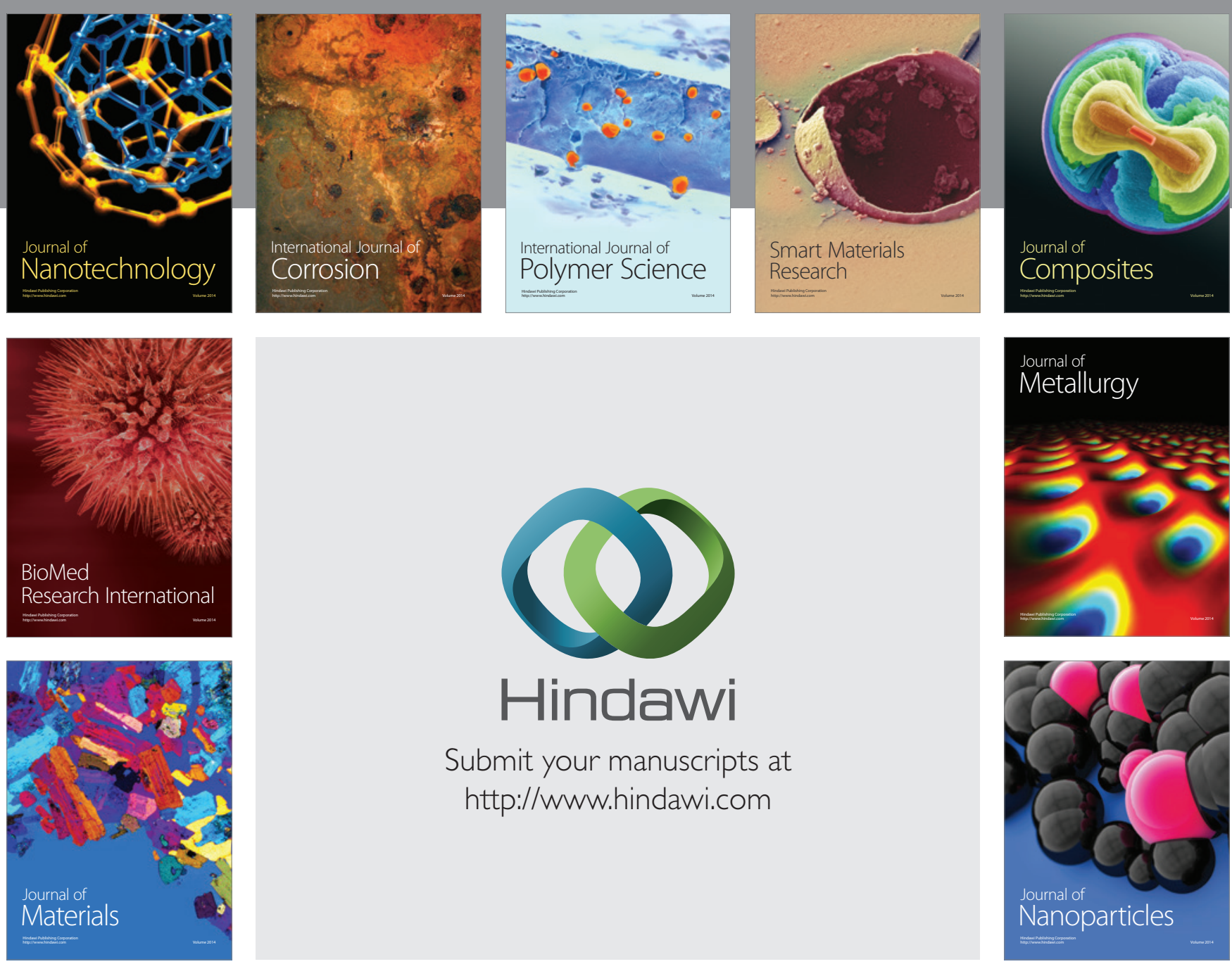

Submit your manuscripts at http://www.hindawi.com
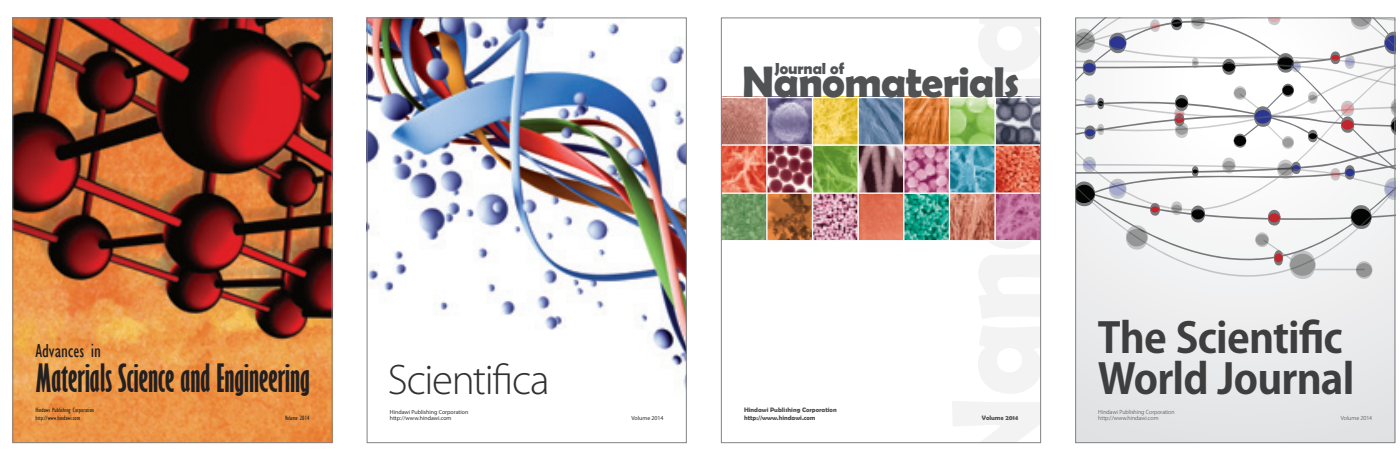

\section{The Scientific World Journal}
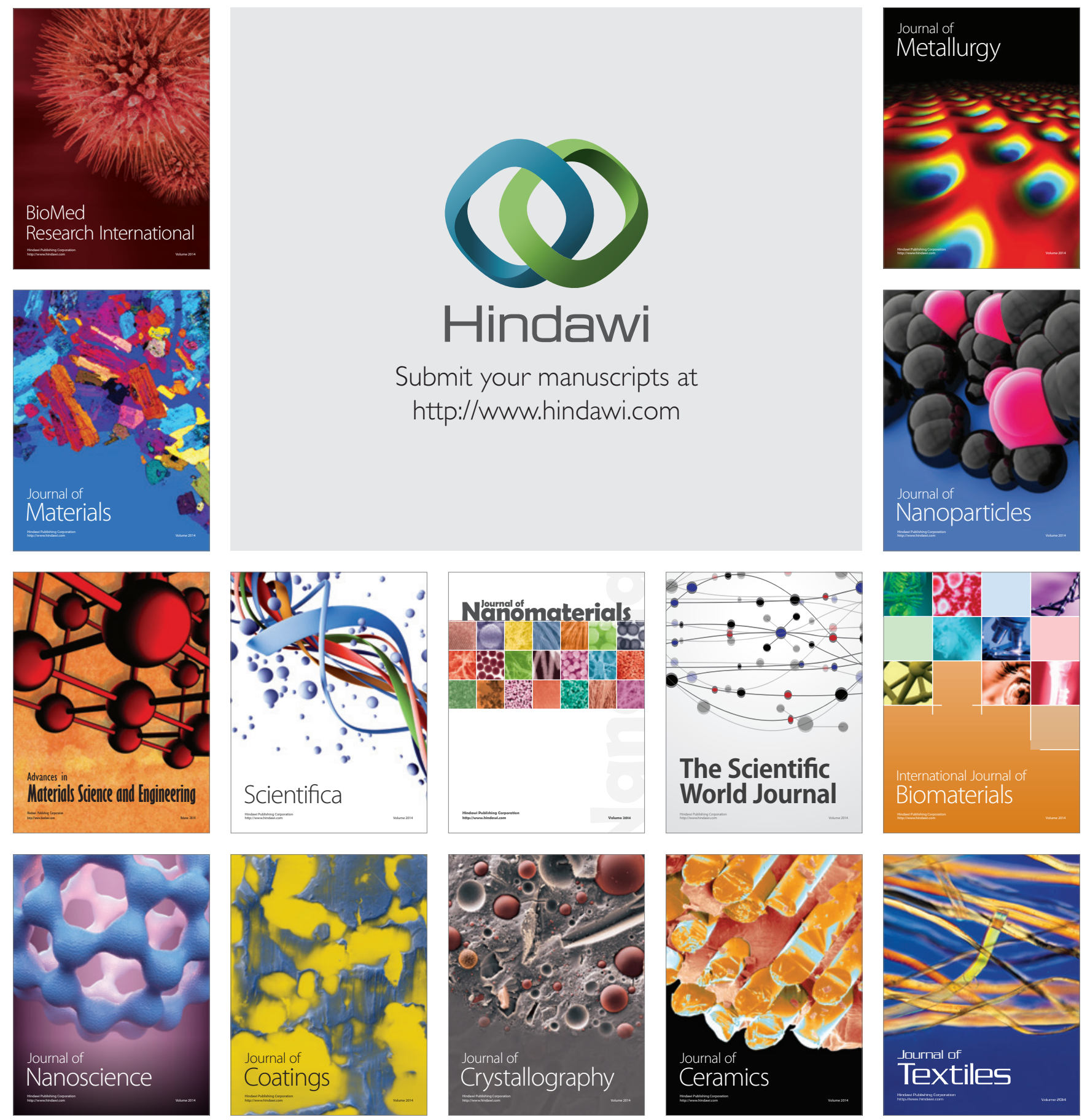